\title{
Evaluation of early implementations of antibiotic stewardship program initiatives in nine Dutch hospitals
}

Maarten van Limburg ${ }^{1 *}$, Bhanu Sinha ${ }^{2}$, Jerome R Lo-Ten-Foe ${ }^{2}$ and Julia EWC van Gemert-Pijnen ${ }^{1}$

\begin{abstract}
Background: Antibiotic resistance is a global threat to patient safety and care. In response, hospitals start antibiotic stewardship programs to optimise antibiotic use. Expert-based guidelines recommend strategies to implement such programs, but local implementations may differ per hospital. Earlier published assessments determine maturity of antibiotic stewardship programs based on expert-based structure indicators, but they disregard that there may be valid deviations from these expert-based programs.
\end{abstract}

Aim: To analyse the progress and barriers of local implementations of antibiotic stewardship programs with stakeholders in nine Dutch hospitals and to develop a toolkit that guides implementing local antibiotic stewardship programs.

Methods: An online questionnaire based on published guidelines and recommendations, conducted with seven clinical microbiologists, seven infectious disease physicians and five clinical pharmacists at nine Dutch hospitals.

Results: Results show local differences in antibiotic stewardship programs and the uptake of interventions in hospitals. Antibiotic guidelines and antibiotic teams are the most extensively implemented interventions. Education, decision support and audit-feedback are deemed important interventions and they are either piloted in implementations at academic hospitals or in preparation for application in non-academic hospitals. Other interventions that are recommended in guidelines - benchmarking, restriction and antibiotic formulary - appear to have a lower priority. Automatic stop-order, pre-authorization, automatic substitution, antibiotic cycling are not deemed to be worthwhile according to respondents.

Conclusion: There are extensive local differences in the implementation of antibiotic stewardship interventions. These differences suggest a need to further explore the rationale behind the choice of interventions in antibiotic stewardship programs. Rather than reporting this rationale, this study reports where rationale can play a key role in the implementation of antibiotic stewardship programs. A one-size-fits-all solution is unfeasible as there may be barriers or valid reasons for local experts to deviate from expert-based guidelines. Local experts can be supported with a toolkit containing advice based on possible barriers and considerations. These parameters can be used to customise an implementation of antibiotic stewardship programs to local needs (while retaining its expert-based foundation).

Keywords: Evaluation, Implementation, Antibiotic stewardship programs, Maturity, Hospital infections

\footnotetext{
* Correspondence: a.h.m.vanlimburg@utwente.nl

'Department Psychology Health and Technology, Faculty Behavioral, Management and Social Sciences, University of Twente, Enschede, the Netherlands

Full list of author information is available at the end of the article
} 


\section{Background}

Antibiotic resistance is an increasing worldwide threat to patient safety and quality of care $[1,2]$. A correlation between antibiotic (over) use and increasing antibiotic resistance is widely acknowledged [3]. Hospitals can influence antibiotic use with improved, prudent antibiotic prescription, as up to $50 \%$ of prescribed antibiotics may be inappropriate or even unnecessary [4]. To curb the increasing resistance, hospitals started antibiotic stewardship programs (ASPs) as quality initiatives for infection prevention and control. An ASP ensures proper use of antibiotics with the best patient outcomes, less risk of adverse effects, optimal cost-effectiveness and to reduce or stabilise levels of resistance [5]. Guidelines from professional societies, such as the Infectious Diseases Society of America/Society for Healthcare Epidemiology of America (IDSA/SHEA) recommend expert-based strategies and interventions to implement ASPs in hospitals [6-8]. In the Netherlands, the Dutch Working Party on Antibiotic Policy (SWAB) is responsible for guideline development, education and surveillance for optimal antibiotic use. This organisation recently published a vision document, stressing the need for Dutch hospitals to form an ASP team and start planning future ASP initiatives as per January 2014 [9].

There is no consistent approach in local implementations of ASP in hospitals [10]. Hospitals combine expertbased strategies and subsequent ASP interventions in many different variations [4]. In a prior systematic review, neither a consistent implementation strategy nor a sufficiently described implementation rationale could be identified in international ASP studies (van Limburg $M$, Köck R, Karreman J, Sinha B, de Jong N, Wentzel J, Friedrich A, Hendrix R, van Gemert-Pijnen J, "Towards an Implementation Strategy for Antibiotic/Antimicrobial Stewardship: A Systematic Review", Under review). To understand how these local implementations of ASP take form it is important to use a bottom-up analysis from within hospitals. In this approach, we involve the relevant local stakeholders in the hospitals we aim to assist with implementing ASP, as opposed to an expert-driven or authoritative top-down approach.

Progression in implementation can be assessed with the concept of 'maturity'. Maturity models were first coined by Paulk et al [11] and are used in many disciplines to model or evaluate improvement of organisations and processes [12]. Maturity models describe a levelled progress of how, in this case, an ASP implementation gradually improves from an ad-hoc state to a structured and managed state and eventually to a measurable and self-optimizing state [11].

Maturity assessments are already used in the context of ASP. Earlier maturity assessments of ASPs were done by the Antibiotic Strategy International group who introduced a comprehensive list of potential structure indicators to assess maturity of ASP in hospitals in several European countries [13]. In short, this means that maturity of ASP depends on the number of expert-based structure indicators that are implemented as part of ASP in a hospital.

This study does not attempt to assess maturity by scoring a completeness of an ideal program, but analyses differences in local implementations of ASP interventions that are recommended in guidelines. Our aim is not primarily to identify which interventions are or should be implemented, but more importantly, to identify where rationale plays a role in implementing ASP interventions and what the consequences are for expert-based ASP strategies. The present study is the first step in our bottom-up assessment with local ASP experts to give an overview of which recommended interventions are implemented, or are being developed, combined with an importance analysis of these interventions. Incongruent responses from stakeholders show where possible difficulties or barriers surface when implementing ASP conform currently available expert guidelines and indicate possibilities for decision support. A follow-up study is required to understand the deeper rationale behind these differences and barriers and how local experts can be assisted with relevant implementation advice.

\section{Methods}

An online questionnaire using LimeSurvey was made available from August to November 2013. Clinical microbiologists, clinical pharmacists and infectious disease physicians were approached as these expert types are identified as being key-stakeholders in ASP (van Limburg M, Köck R, Karreman J, Sinha B, de Jong N, Wentzel J, Friedrich A, Hendrix R, van Gemert-Pijnen J, "Towards an Implementation Strategy for Antibiotic/Antimicrobial Stewardship: A Systematic Review", Under review). Ethical approval was given after revision by an internal representative of the ethical board (reference: BCE14039) and no further ethical review procedures were deemed necessary. We sent a questionnaire invitation to known infection control experts recruited in earlier research projects. These experts represented academic and nonacademic hospitals in the Dutch eastern border region and they were asked to distribute the questionnaire among clinical microbiologists, clinical pharmacists and infectious disease physicians tasked with ASP in their hospitals. As at the time of the questionnaire ASP teams were not necessarily formed at the hospitals yet, we chose to approach key stakeholders rather than teams directly. We also analysed the response of key stakeholders individually (not clustered per hospital) as stakeholders may have possible differences in views on ASP interventions (even within hospitals). 
The questionnaire was structured around ten implementation topics based on a literature review (see Table 1). The topics that were addressed involved ASP program initiatives, presence and importance of commonly used interventions and their possible characteristics, and possible outcomes and effects relevant for assessing evidence for the effectiveness of ASP. The extraction of implementation topics concerning ASP maturity or implementation assessments, attitude or opinion surveys towards ASP or practical implementation toolkits for ASP are presented in detail in Table 1.

The final questionnaire was adapted for the Dutch healthcare context, reduced and validated by microbiologists to have a final scope of 47 questions for an acceptable answering duration of 15-20 minutes. A literal English translation of the Dutch questionnaire can be found in Additional file 1 for reference.

Responses were imported into SPSS21 for statistical analysis. Incomplete responses were excluded. Frequency cross-tables of responses stratified by hospital type (academic/non-academic) were used for reporting all results, as we anticipated differences between the two hospital types [10].

\section{Results}

\section{Response}

A total of 27 responses were collected. Eight respondents started answering the first few questions but did not complete the questionnaire and had to be excluded from the results. Based on the first questions we could identify that these were three clinical microbiologists, one infectious disease physician and four clinical pharmacists. Nineteen respondents completed the questionnaire, and the final sample consists of seven clinical microbiologists, seven infectious disease physicians and five clinical pharmacists from three academic hospitals and six non-academic (general tertiary care) hospitals.

\section{Antibiotic stewardship program initiatives}

The three included academic hospitals have implemented an ASP. At the non-academic hospitals, ASP initiatives are in development at five hospitals while one hospital has implemented an ASP. Almost all ASP initiatives were started from 2012 onwards.

Respondents from academic hospitals seemed more satisfied with current mandate from hospital management, available budgets and assigned full-time equivalents (FTEs) than their non-academic peers. No consensus was found in their opinions regarding satisfaction with the level of formalisation of ASP with, for example, documentation or task descriptions.

Academic hospitals targeted multiple wards in their ASP implementations, but the type of wards varied. Generally these were all wards with high risk of infections and with relatively high antibiotic use. Most non-academic hospitals did not yet implement ASP. There, however, is a strong consensus that eventually ASPs should be implemented throughout the entire hospital.

\section{Implementation of ASP interventions}

Table 2 provides an overview of the maturity assessment as to whether common ASP interventions were already implemented, in development, considered necessary or considered unnecessary. The following paragraphs discuss the interventions in more detail.

\section{Antibiotic team}

In the early stages of ASP implementation, an antibiotic team (usually called A-team for obvious reasons) is mostly responsible for preparing an ASP. Subsequently, their tasks change to monitoring ASP effectiveness and performing important tasks that are part of the program. Based on the response, we can assume antibiotic teams were either already formed or in preparation.

Table 3 shows the composition of these teams. In both types of hospitals the antibiotic teams consist of at least infectious disease physicians, clinical microbiologists and clinical pharmacists. These are also the stakeholders recommended to be at least present according to the national guidelines endorsed by SWAB [9]. Other stakeholders are involved in different team configurations but there is no consensus on which of these other stakeholders should be definite members of the antibiotic team. In both types of hospitals it is clear that management and attending physicians have no active role in the team.

In non-academic hospitals, the frequency of infectious disease physicians in the teams is lower compared to academic hospitals. This can be explained by the probability that non-academic hospitals have no or too few (e.g. shared with other local hospitals) infectious disease physicians available, hence they cannot take part in their antibiotic team.

\section{(Local) Antibiotic guidelines}

There are national antibiotic guidelines to which Dutch hospitals have to comply, based on evidence or expertbased guidelines and policies established in academic hospitals (e.g. available at www.swabid.nl). Hospitals are at liberty to use these national guidelines in full or adapt them slightly into local antibiotic guidelines.

Table 4 shows that academic hospitals have most common types of antibiotic guidelines locally implemented and overall, respondents were satisfied with currently available antibiotic guidelines in their hospitals. They responded that guidelines for intravenous-per os switches and for deescalation are not yet available, but needed.

The situation in non-academic hospitals is different. Guidelines for treatment of infections, antibiotic therapy 
Table 1 Overview of literature scan and extracted implementation topics

\begin{tabular}{ll}
$\#$ & Author $(\mathbf{s})$ \\
\hline $\mathbf{1}$ & Bannan, Buono, McLaws,
\end{tabular} Gottlieb

2
3* Burgmann, Janata, Allerberger, Frank
Year Title

2009 Survey of medical staff attitudes to an antibiotic approval and stewardship program
Study description

Design:

Questionnaire with 40 questions focused on restriction and approval

Interest:

Attitude

2006 Antibiotic-stewardship practices at top academic centers throughout the united states and at hospitals throughout Massachusetts
Design:

Two surveys

Interest:

ASP components
Extracted implementation topics

- restriction as intervention

- authorization as intervention

- advice as communication

- education as intervention

- stop-order (withholding pharmacy) as intervention

- costs, appropriate use, resistance, time as outcomes

- pager as communication

- possible stakeholders in team

- multifaceted programs

- time of start with ASP

- funding/financial support

- (formulary) restriction as intervention

- solicited input from ID as communication

- costs, improved use, adverse effects, resistance, compliance, DDDs, clinical outcomes as outcomes

- aiming prophylaxis

- aiming only targeted antibiotics

- aiming antibiotic therapy at order

- aiming initial therapy

- recommendations as intervention (day 3 bundle)

- culture data as communication

- possible stakeholders in team

- approval as intervention

- review as communication

- consult as communication

- computerized order entry as communication

- stop-order as intervention

- IV-PO switch as intervention

- clinical practical guidelines as intervention

- evaluation as intervention (benchmarking)

- support and time needed from physicians

- rounds, didactics, program, consults/feedback as education

2008 Hospital antibiotic management Design: in Austria - results of the ABS maturity survey of the ABS International group
- data evaluation as intervention (benchmarking)

- AB consumption data as outcomes 
Table 1 Overview of literature scan and extracted implementation topics (Continued)

\begin{tabular}{|c|c|c|c|c|c|}
\hline & & & & Interest: & $\begin{array}{l}\text { - hospital/department/ward levels } \\
\text { of benchmarking }\end{array}$ \\
\hline & & & & 5 categories of maturity & $\begin{array}{l}\text { - feedback of benchmarking as } \\
\text { communication }\end{array}$ \\
\hline & & & & & - possible stakeholders in team \\
\hline & & & & & $\begin{array}{l}\text { - guidelines for dosage, drug costs, } \\
\text { IV-PO switch }\end{array}$ \\
\hline & & & & & - guidelines for antibiotic treatment \\
\hline & & & & & - guidelines for prophylaxis \\
\hline & & & & & $\begin{array}{l}\text { - education as intervention } \\
\text { (seminars, literature) }\end{array}$ \\
\hline & & & & & - financial resources \\
\hline & & & & & - cooperation with other hospitals \\
\hline \multirow[t]{19}{*}{4} & \multirow{19}{*}{$\begin{array}{l}\text { Buyle, Metz-Gercek, Mechtler, } \\
\text { Kern, Robays, Vogelaers, } \\
\text { Struelens }\end{array}$} & \multirow[t]{19}{*}{2013} & \multirow{19}{*}{$\begin{array}{l}\text { Development and validation of } \\
\text { potential structure indicators } \\
\text { for evaluating antimicrobial } \\
\text { stewardship programmes in } \\
\text { European hospitals }\end{array}$} & Design: & - bedside advice as communication \\
\hline & & & & Expert panel + validation survey & - rounds as intervention \\
\hline & & & & & - frequency of team meetings \\
\hline & & & & Interest: & - audit as intervention \\
\hline & & & & Potential structure indicators & - possible stakeholders in team \\
\hline & & & & & - formulary as intervention \\
\hline & & & & & - updating formulary \\
\hline & & & & & - stop order as intervention \\
\hline & & & & & $\begin{array}{l}\text { - guidelines for microbiological } \\
\text { documented therapy, empirical } \\
\text { therapy, prophylaxis, iv-po } \\
\text { switches }\end{array}$ \\
\hline & & & & & - updating guidelines \\
\hline & & & & & - clinical decision aid as IT \\
\hline & & & & & - mandate from management \\
\hline & & & & & - FTEs \\
\hline & & & & & - Education as interventions \\
\hline & & & & & $\begin{array}{l}\text { - passive methods, interactive } \\
\text { methods as education }\end{array}$ \\
\hline & & & & & - evaluation as intervention \\
\hline & & & & & - resistance data, consumption data, \\
\hline & & & & & $\begin{array}{l}\text { - hospital/department/ward levels } \\
\text { of benchmarking }\end{array}$ \\
\hline & & & & & $\begin{array}{l}\text { - total DDDs, \# of infections as } \\
\text { outcomes }\end{array}$ \\
\hline \multirow[t]{9}{*}{5} & \multirow{9}{*}{$\begin{array}{l}\text { Cooke, Alexander, Charani, } \\
\text { Hand, Hills, Howard, Jamieson, } \\
\text { Lawson, Richardson, Wade }\end{array}$} & \multirow[t]{9}{*}{2010} & \multirow{9}{*}{$\begin{array}{l}\text { Antimicrobial stewardship: an } \\
\text { evidence-based, antimicrobial } \\
\text { self-assessment toolkit (ASAT) } \\
\text { for acute hospitals }\end{array}$} & Design: & - guidelines as intervention \\
\hline & & & & ASAT toolkit (checklist) & - formulary as intervention \\
\hline & & & & Interest: & - restriction as intervention \\
\hline & & & & Levels of antimicrobial & - IV-PO switches as intervention \\
\hline & & & & stewardship & $\begin{array}{l}\text { - guidelines for prophylaxis as } \\
\text { intervention }\end{array}$ \\
\hline & & & & & - adherence as outcome \\
\hline & & & & & - education as intervention \\
\hline & & & & & - training as education \\
\hline & & & & & - information systems as IT \\
\hline
\end{tabular}


Table 1 Overview of literature scan and extracted implementation topics (Continued)

6 Dumartin, Rogues, Amadeo, Pefau, Venier, Parneix, Maurain

7 van Gastel, Costers, Peetermans, Struelens

8 Greater New York Hospital Association
2011 Antibiotic stewardship programmes: legal framework and structure and process indicator in Southwestern French hospitals, 2005-2008

Design:
Survey
Interest:

Checking whether legal framework is present

Design:

Questionnaire antibiotic management teams in Belgian hospitals: a self-reporting survey

Interest:

Level of AMT activities

Design:

Best practice

Interest:

Implementation toolkit
- digital prescribing as IT

- possible stakeholders in team

- frequency in meetings

- available human resources

- digital prescription, pharmaceutical analysis, dispensation, digital link between lab, pharm, wards as IT

- restriction as intervention

- stop order as intervention

- first-line, prophylaxis as guidelines

- audits as intervention/ communication

- evaluation feedback as communication

- education as intervention

- Formulary as intervention

- ab consumption as benchmarking

- DDDs, resistance as outcomes (and communication)

- possible stakeholders in team

- Possible stakeholders in team

- consultation per phone, email, intranet, face-to-face, staff meetings as communication

- formulary as intervention

- guidelines for empirical and prophylaxis

- updates of formulary and guidelines

- restriction as intervention

- approval/review as intervention

- concurrent review/audit as intervention

- de-escalation as intervention

- stop order as intervention

- order forms as intervention

- IV-PO switch as intervention

- consumption and resistance as outcomes

- by hospital/unit or by antibiotic type

- feedback of outcomes

- benchmark and review antibiotic use (patterns)

- review resistance

- IT infrastructure

- possible stakeholders in team

- aim for common infections, pathogens, agents

- rollout: hospital vs. ward 
Table 1 Overview of literature scan and extracted implementation topics (Continued)

9 Hulscher, Grol, van der Meer

10 Nault, Beaudoin, Thirion, Gosselin, Cossette, Valiquette
2010 Antibiotic prescribing in hospitals: a social and behavioral scientific approach

2008 Antimicrobial stewardship in acute care centres: survey of 68 hospitals in Quebec
- available resources

- strategy:

- guidelines for diagnosis, treatment, duration, dose optimization, IV-PO, streamlining/de-escalation

- formulary as intervention

- restriction as intervention

- education as intervention

- prospective review as intervention

- stickers, notes, face-to-face as communication

- data collection (benchmarking)

- usage, clinical, microbiologic costs as data

- formulary as intervention

- order form as intervention

- restriction as intervention

Interest:

socio-cultural factors of ASP

- stop orders as intervention

- infection control committee

- guidelines as intervention

- review as intervention

- rounds as intervention

- telephone advice as intervention

- improve infrastructure

- education as intervention

- conferences, seminars, skill training programs as education

- individual instructions (outreach, academic detailing)

- feedback of outcomes

- decision support via IT

Design:

Questionnaire

Interest:

Proportion and nature of programs
- Duration of ASP or busy setting up

- distributed units, DDDs, acquisition costs as benchmarking data

- direct interaction as intervention (written or phone)

- education as intervention

- stop orders as intervention

- auto substitution

- formulary restriction as interventions

- local guidelines as intervention

- preauthorization as intervention

- antibiotic cycling as intervention

- decision support systems as intervention

- possible stakeholders in team 
Table 1 Overview of literature scan and extracted implementation topics (Continued)

\begin{tabular}{|c|c|c|c|c|c|}
\hline \multirow[t]{10}{*}{11} & \multirow{10}{*}{$\begin{array}{l}1 \text { Pulcini, Williams, Molinari, } \\
\text { Davey, Nathwani }\end{array}$} & \multirow[t]{10}{*}{2011} & \multirow{10}{*}{$\begin{array}{l}\text { Junior doctors' knowledge and } \\
\text { perceptions of antibiotic resistance } \\
\text { and prescribing: a survey in France } \\
\text { and Scotland }\end{array}$} & Design: & - local guidelines as intervention \\
\hline & & & & Survey & - presence of team- \\
\hline & & & & Interest: & - approval as intervention \\
\hline & & & & \multirow{7}{*}{$\begin{array}{l}\text { Perception and prescribing } \\
\text { practice }\end{array}$} & - IV-PO switch protocol \\
\hline & & & & & $\begin{array}{l}\text { - advice from ID physician, senior, } \\
\text { microbiologist, pharmacist or } \\
\text { team as intervention }\end{array}$ \\
\hline & & & & & $\begin{array}{l}\text { - face-to-face, phone, consult upon } \\
\text { request as communication }\end{array}$ \\
\hline & & & & & $\begin{array}{l}\text { - lectures, workshops, informal } \\
\text { education, web-based learning, } \\
\text { self-directed learning as education }\end{array}$ \\
\hline & & & & & - possible stakeholders in team \\
\hline & & & & & - computer aided prescribing as IT \\
\hline & & & & & - resistance data availability \\
\hline \multirow[t]{11}{*}{12} & \multirow[t]{11}{*}{ Thern } & \multirow[t]{11}{*}{2013} & $\begin{array}{l}\text { Selection of hospital antimicrobial } \\
\text { prescribing quality indicators: }\end{array}$ & Design: & - possible stakeholders in team \\
\hline & & & \multirow{10}{*}{$\begin{array}{l}\text { a consensus among German } \\
\text { antibiotic stewardship (ABS) } \\
\text { networkers }\end{array}$} & Review+ & - frequency of meetings \\
\hline & & & & questionnaire & - mandate \\
\hline & & & & Interest: & - drug use, resistance rates as data \\
\hline & & & & \multirow{7}{*}{$\begin{array}{l}\text { Indicators for quality of } A B \\
\text { prescribing }\end{array}$} & - formulary as intervention \\
\hline & & & & & - updating formulary \\
\hline & & & & & - restriction/approval as intervention \\
\hline & & & & & $\begin{array}{l}\text { - guidelines for empiric therapy, } \\
\text { IV-PO, dosing, prophylaxis, }\end{array}$ \\
\hline & & & & & - rounds as intervention \\
\hline & & & & & - education as intervention \\
\hline & & & & & $\begin{array}{l}\text { - guidance or assisted decision } \\
\text { analysis via IT }\end{array}$ \\
\hline \multirow[t]{16}{*}{13} & \multirow[t]{16}{*}{ Trivedi, Rosenberg } & \multirow[t]{16}{*}{2013} & \multirow{16}{*}{$\begin{array}{l}\text { The state of antimicrobial } \\
\text { stewardship programs in } \\
\text { California }\end{array}$} & Design: & - implemented or planned ASP \\
\hline & & & & Survey & - time of start with ASP \\
\hline & & & & Interest: & - possible stakeholders in team \\
\hline & & & & \multirow[t]{13}{*}{ State of ASP } & - FTE availability \\
\hline & & & & & - - funding \\
\hline & & & & & - benchmarking as intervention \\
\hline & & & & & $\begin{array}{l}\text { - DDDs, DOTs, costs, acceptance } \\
\text { of recommendations, improved } \\
\text { susceptibility patterns as data }\end{array}$ \\
\hline & & & & & - use of IT in ASP \\
\hline & & & & & $\begin{array}{l}\text { - electronic health record, digital } \\
\text { prescription, electronic medication } \\
\text { administration records as IT }\end{array}$ \\
\hline & & & & & - formulary restriction as intervention \\
\hline & & & & & - ID physician consult as intervention \\
\hline & & & & & - audit as intervention \\
\hline & & & & & - prior approval as intervention \\
\hline & & & & & - auto stop orders as intervention \\
\hline & & & & & - verbal approval as intervention \\
\hline & & & & & - pre-authorization as intervention \\
\hline
\end{tabular}




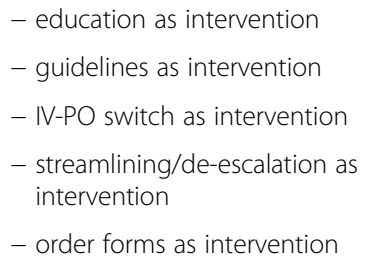

- order forms as intervention

and prophylaxis are implemented (these are all available as national antibiotic guidelines). However, other antibiotic guidelines are incomplete and not as available when compared to academic hospitals. Respondents from non-academic hospitals agree that additional antibiotic guidelines are needed. It is interesting to see that exactly those guidelines that academic hospitals expressed as being needed (intravenous-per os switches and de-escalation) are in development at non-academic hospitals.

\section{Antibiotic formulary}

An antibiotic formulary contains an overview of indications and favourable antibiotic treatment (s). In the Netherlands, these formularies are usually introduced and maintained by the clinical microbiologists. Overall, an antibiotic formulary is present in both types of hospitals.

\section{Audit-and-feedback}

Audits are interactions with prescribers to influence the way they prescribe for antibiotic therapy. These audits can be prospective, thus directly influence therapy with feedback at patient level, or retrospective where the prescribed antibiotics are assessed and reported back to prescribers.

The response (see Table 2) from respondents at academic hospitals is diverse and suggests audits are performed but still as a pilot in only a few wards. As Figure 1 depicts, there is no clear preference for bedside or remote consults as form of audit, but retrospective feedback seems less preferred.

At non-academic hospitals audits do not yet take place but are in either in development or considered necessary. Compared to academic hospitals the respondents of non-academic hospitals have a slightly stronger preference for bedside audits over remote audits via phone or email as can be seen in Figure 1. All respondents stated audit-and-feedback was the preferred strategy for ASP.

\section{Education}

Educational activities are needed to disseminate the goals and interventions of ASP among prescribers and other possible stakeholders for ASP. At academic hospitals, educational activities implemented or in development relatively equally, suggesting it this is something that is

Table 2 Maturity of ASP interventions in academic and non-academic hospitals

\begin{tabular}{|c|c|c|c|c|c|c|c|c|c|c|}
\hline & \multicolumn{5}{|c|}{ Academic hospitals } & \multicolumn{5}{|c|}{ Non-academic hospitals } \\
\hline & Impl & In dev & Need & Unneed & $N / A^{*}$ & Impl & In dev & Need & Unneed & $N / A^{*}$ \\
\hline Antibiotic team & $6(75 \%)$ & $2(25 \%)$ & & - & - & $6(55 \%)$ & $5(45 \%)$ & - & - & - \\
\hline (Local) antibiotic guidelines & $7(88 \%)$ & $1(12 \%)$ & - & - & - & $9(82 \%)$ & $2(18 \%)$ & - & - & - \\
\hline Antibiotic formulary & $7(88 \%)$ & $1(13 \%)$ & - & - & - & $8(73 \%)$ & $2(18 \%)$ & $1(9 \%)$ & - & - \\
\hline Audit-and-feedback & $3(38 \%)$ & $2(25 \%)$ & $2(25 \%)$ & - & $1(13 \%)$ & - & $4(36 \%)$ & $6(55 \%)$ & $1(9 \%)$ & - \\
\hline Education & $4(50 \%)$ & $4(50 \%)$ & - & - & - & $2(18 \%)$ & $8(73 \%)$ & $1(9 \%)$ & - & - \\
\hline Information systems for ASP & $2(25 \%)$ & $1(13 \%)$ & $5(63 \%)$ & - & - & $2(18 \%)$ & $4(36 \%)$ & $3(27 \%)$ & - & $2(18 \%)$ \\
\hline Benchmarking & $4(50 \%)$ & $3(38 \%)$ & - & $1(13 \%)$ & - & $1(9 \%)$ & $7(64 \%)$ & $2(18 \%)$ & - & $1(9 \%)$ \\
\hline Restriction & $2(25 \%)$ & $4(50 \%)$ & $1(13 \%)$ & $1(13 \%)$ & & & $5(45 \%)$ & $3(27 \%)$ & $1(9 \%)$ & $2(18 \%)$ \\
\hline Academic detailing & $1(13 \%)$ & $3(38 \%)$ & $3(38 \%)$ & - & $1(13 \%)$ & $2(18 \%)$ & $2(18 \%)$ & $4(36 \%)$ & - & $3(27 \%)$ \\
\hline Automatic stop-order & - & $1(13 \%)$ & $4(50 \%)$ & $2(25 \%)$ & $1(13 \%)$ & - & $4(36 \%)$ & $3(27 \%)$ & $2(18 \%)$ & $2(18 \%)$ \\
\hline Pre-authorization & $1(13 \%)$ & $1(13 \%)$ & $3(38 \%)$ & $1(13 \%)$ & $2(25 \%)$ & - & $2(18 \%)$ & $3(27 \%)$ & $1(9 \%)$ & $5(45 \%)$ \\
\hline Automatic substitution & - & $1(13 \%)$ & $2(25 \%)$ & $5(63 \%)$ & & - & 1 (9\%) & $3(27 \%)$ & $2(18 \%)$ & $5(45 \%)$ \\
\hline Antibiotic cycling & - & $1(13 \%)$ & $1(13 \%)$ & $4(50 \%)$ & $2(25 \%)$ & - & - & $5(45 \%)$ & $2(18 \%)$ & $4(36 \%)$ \\
\hline
\end{tabular}

*) Impl: implemented; in dev: in development; need: are needed; unneed: are unneeded; N/A: no answer or not applicable. 
Table 3 Composition of antibiotic team in academic and non-academic hospitals

\begin{tabular}{|c|c|c|c|c|c|c|c|c|c|c|}
\hline & \multicolumn{5}{|c|}{ Academic hospitals } & \multicolumn{5}{|c|}{ Non-academic hospitals } \\
\hline & Impl & In dev & Need & Unneed & $N / A^{*}$ & Impl & In dev & Need & Unneed & $N / A^{*}$ \\
\hline Clinical microbiologist & $8(100 \%)$ & $x$ & & - & - & $9(82 \%)$ & $x$ & $1(9 \%)$ & - & $1(9 \%)$ \\
\hline Infectious disease physician & $8(100 \%)$ & $x$ & & - & - & $5(45 \%)$ & $x$ & - & $1(9 \%)$ & $5(45 \%)$ \\
\hline Clinical pharmacist & 7 (88\%) & $x$ & & $1(13 \%)$ & - & $9(82 \%)$ & $x$ & $1(9 \%)$ & - & $1(9 \%)$ \\
\hline Member of antibiotic committee & $5(63 \%)$ & $x$ & $1(13 \%)$ & - & $2(25 \%)$ & $4(36 \%)$ & $x$ & $3(27 \%)$ & $1(9 \%)$ & $3(27 \%)$ \\
\hline Prescribing physician & $4(50 \%)$ & $x$ & - & $3(38 \%)$ & $1(13 \%)$ & - & $x$ & $4(36 \%)$ & $3(27 \%)$ & $4(36 \%)$ \\
\hline Hygienist & $1(13 \%)$ & $x$ & $3(38 \%)$ & $3(38 \%)$ & $1(13 \%)$ & $1(9 \%)$ & $x$ & $3(27 \%)$ & $4(36 \%)$ & $3(27 \%)$ \\
\hline IT specialist & - & $x$ & $3(38 \%)$ & $4(50 \%)$ & $1(13 \%)$ & - & $x$ & $4(36 \%)$ & $5(45 \%)$ & $2(18 \%)$ \\
\hline Nurse & - & $x$ & $3(38 \%)$ & $3(38 \%)$ & $2(25 \%)$ & - & $x$ & $2(18 \%)$ & $5(45 \%)$ & $4(36 \%)$ \\
\hline Epidemiologist & $1(13 \%)$ & $x$ & $4(50 \%)$ & $1(13 \%)$ & $2(25 \%)$ & - & $x$ & $1(9 \%)$ & $4(36 \%)$ & $6(55 \%)$ \\
\hline Management & - & $x$ & - & $6(75 \%)$ & $1(13 \%)$ & - & $x$ & $2(18 \%)$ & $6(55 \%)$ & $2(18 \%)$ \\
\hline Supervising physician & $1(13 \%)$ & $x$ & $1(13 \%)$ & $5(63 \%)$ & $1(13 \%)$ & - & $x$ & $1(9 \%)$ & $6(55 \%)$ & $4(36 \%)$ \\
\hline
\end{tabular}

*) Impl: implemented; in dev: in development; need: are needed; unneed: are unneeded; N/A: no answer or not applicable.

being piloted and still taking shape. Non-academic hospitals have progressed less with implementing education and hence educational activities are still contemplated or in development. Respondents of both types of hospitals have a strong preference for workshops as means of education (Figure 2).

\section{Information systems for ASP}

Specific information systems (information technology or software) that can be used in ASP were present at academic hospitals, except for information systems to evaluate prescriptions and decision support tools, e.g. for information while performing audits.

At non-academic hospitals, the presence of information systems was more divided than at their academic peers and not generalizable, but information systems for ASP are mostly in development Table 5.

\section{Benchmarking}

Benchmarking or monitoring antibiotic use related outcomes is necessary to assess changes caused by ASP.
Such data can be compared internally (e.g. over time, between wards, between prescribers, etc.) and externally (e.g. between hospitals, nationally, internationally, etc.). Antibiotic teams at academic hospitals that benchmark or have it in development have access to common data sources for benchmarking. In non-academic hospitals benchmarking is less present and still in development, hence most data sources are not (yet) available either to the antibiotic team. Figure 3 gives an overview of the available (or in case of non-academic hospitals, in development) data sources. The figure suggests that daily defined doses and antibiotic costs are relatively the most used data types for benchmarking and monitoring antibiotic use.

\section{Restriction}

Restriction of antibiotics means prescription of some or all antibiotics requires validation by a member of the antibiotic team. This intervention is not commonly present in academic hospitals, but stated as being in development. Current and future application of

Table 4 Status of antibiotic guidelines in academic and non-academic hospitals

\begin{tabular}{|c|c|c|c|c|c|c|c|c|c|c|}
\hline & \multicolumn{5}{|c|}{ Academic hospitals } & \multicolumn{5}{|c|}{ Non-academic hospitals } \\
\hline & Impl & In dev & Need & Unneed & $N / A^{*}$ & Impl & In dev & Need & Unneed & $N / A^{*}$ \\
\hline Diagnosis of infections & $8(100 \%)$ & - & - & - & - & $4(36 \%)$ & - & $3(27 \%)$ & - & $4(36 \%)$ \\
\hline Treatment of infections & $8(100 \%)$ & - & - & - & - & $7(64 \%)$ & $2(18 \%)$ & - & - & $2(18 \%)$ \\
\hline Antibiotic therapy & $8(100 \%)$ & - & - & - & - & $10(91 \%)$ & $1(9 \%)$ & - & - & - \\
\hline Duration of therapy & $7(88 \%)$ & - & $1(13 \%)$ & - & - & $5(45 \%)$ & $2(18 \%)$ & $3(27 \%)$ & - & - \\
\hline Prophylaxis & $8(100 \%)$ & - & - & - & - & $10(91 \%)$ & $1(9 \%)$ & - & - & - \\
\hline IV-PO switches & $5(63 \%)$ & - & $3(38 \%)$ & - & - & $3(27 \%)$ & $4(36 \%)$ & $4(36 \%)$ & - & - \\
\hline De-escalation/streamlining & $3(38 \%)$ & $1(13 \%)$ & $4(50 \%)$ & - & - & $1(9 \%)$ & $4(36 \%)$ & $5(45 \%)$ & - & - \\
\hline
\end{tabular}

*) Impl: implemented; in dev: in development; need: are needed; unneed: are unneeded; N/A: no answer or not applicable. 


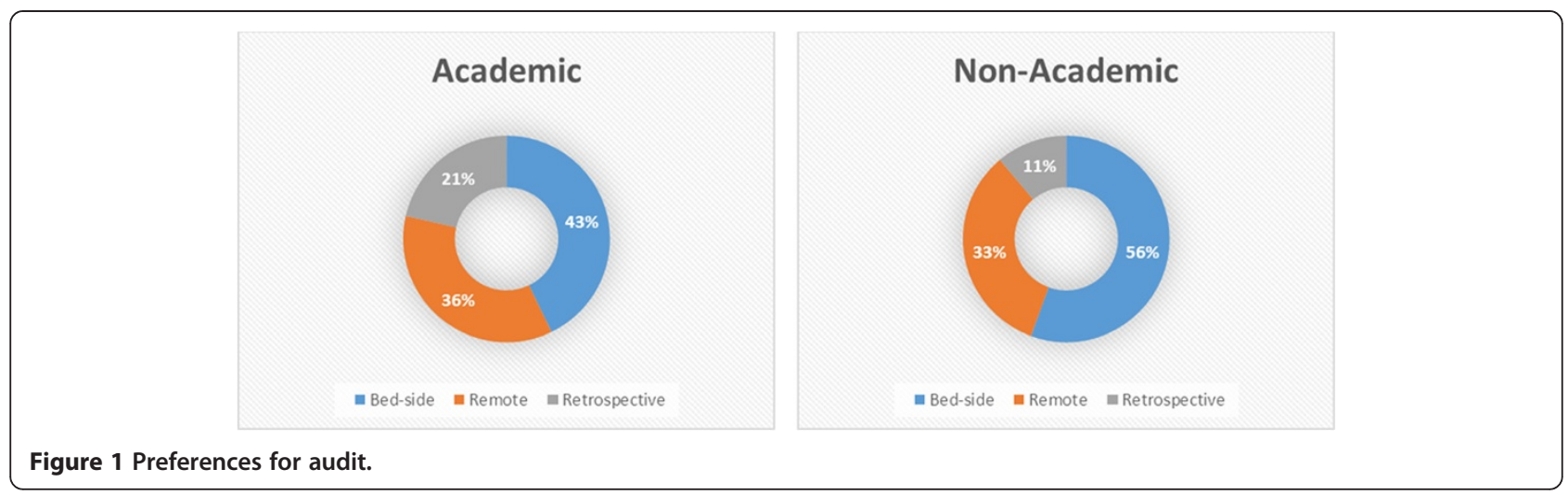

restrictive measures is more common in non-academic hospitals. None of the respondents indicated that restriction and (pre)-approval were identified as key strategies for ASP.

\section{Academic detailing}

Academic detailing means that prescribers are influenced with structural evidence-based feedback, where the academic evidence plays an important role in the feedback as a form of education. This is hardly used in both types of hospitals but respondents stated this intervention is in either development or needed in the longer term. Currently there can be some ad-hoc academic explanation in feedback, but 'academic detailing' as a structural intervention that structurally provides prescribers with evidence-based feedback, is not seen as an important, urgent intervention according to our respondents.

\section{Automatic stop-order, pre-authorisation, automatic substitution and antibiotic cycling}

Respondents of non-academic hospitals stated that these four interventions are all in development or needed. Academic hospitals appear to be only interested in developing automatic stop-orders or pre-authorisation as automatic substitution and antibiotic cycling were often stated as being unnecessary.

\section{Importance of ASP interventions}

Respondents were asked in the questionnaire to rank ASP interventions. The list contained thirteen ASP interventions respondents could rank in order of importance to their ASP with a 1 to 13 score. Final scores in Figure 4 were averaged per type of hospital and overall average. The median of 7 was plotted to emphasize the perceived relative importance of interventions.

Local antibiotic guidelines and an antibiotic team are scored as the two currently most important interventions for implementing ASP in both types of hospitals. Education and audit-and-feedback scored relatively high as well, and in combination with earlier data in Table 2, they can be seen as part of a primary ASP bundle. Decision support systems receive more attention in nonacademic hospitals than in academic hospitals. A possible explanation for this is the higher preference for bedside audits in non-academic hospitals (see Table 2) since for audits such decision support systems are particularly helpful. Less direct attention is needed for restrictive measures, an antibiotic formulary, academic detailing and benchmarking. We think an explanation for the low score for antibiotic formularies is that most Dutch hospitals already have such formularies in place and as such they are not directly seen as a specific, important intervention of ASP. The remaining interventions, automatic stop-order,
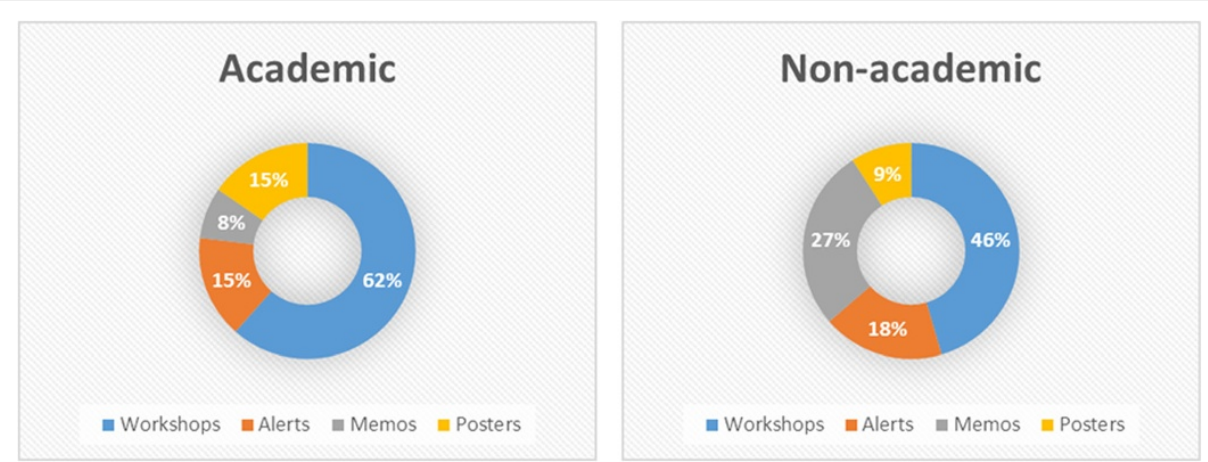

Figure 2 Preferences for education. 
Table 5 Status of information systems for ASP in academic and non-academic hospitals

\begin{tabular}{|c|c|c|c|c|c|c|c|c|c|c|}
\hline & \multicolumn{5}{|c|}{ Academic hospitals } & \multicolumn{5}{|c|}{ Non-academic hospitals } \\
\hline & Impl & In dev & Need & Unneed & $N / A^{*}$ & Impl & In dev & Need & Unneed & $N / A^{*}$ \\
\hline Electronic health records & $5(63 \%)$ & $2(25 \%)$ & $1(13 \%)$ & - & - & $9(82 \%)$ & $2(18 \%)$ & - & - & - \\
\hline Digital laboratory data & $6(75 \%)$ & $1(13 \%)$ & $1(13 \%)$ & - & - & $6(55 \%)$ & $1(9 \%)$ & $3(27 \%)$ & - & $1(9 \%)$ \\
\hline Digital antibiotic use data & 7 (88\%) & - & - & - & $1(13 \%)$ & $4(36 \%)$ & $3(27 \%)$ & $2(18 \%)$ & - & $2(18 \%)$ \\
\hline Digital precribing & $8(100 \%)$ & - & - & - & - & $7(64 \%)$ & $3(27 \%)$ & $1(9 \%)$ & - & - \\
\hline Evaluation of prescription & $2(25 \%)$ & $4(50 \%)$ & $2(25 \%)$ & - & - & $1(9 \%)$ & $2(18 \%)$ & $5(45 \%)$ & - & $3(27 \%)$ \\
\hline Decision support systems & $1(13 \%)$ & $2(25 \%)$ & $3(38 \%)$ & - & $2(25 \%)$ & $1(9 \%)$ & $4(36 \%)$ & $4(36 \%)$ & $1(9 \%)$ & $1(9 \%)$ \\
\hline Surveillance & $6(75 \%)$ & 1 (13\%) & & - & 1 (13\%) & $3(27 \%)$ & $4(36 \%)$ & $4(36 \%)$ & - & - \\
\hline
\end{tabular}

*) Impl: implemented; in dev: in development; need: are needed; unneed: are unneeded; N/A: no answer or not applicable.

pre-authorisation, automatic substitution, and antibiotic cycling score relative low on importance and it is questionable whether these are interesting interventions for ASP.

\section{Discussion}

Both academic hospitals and non-academic hospitals in the Dutch border region are busy with antibiotic stewardship initiatives. When assessing local implementations of ASPs, a clear difference in academic and non-academic hospitals can be seen. Generally, academic hospitals are experimenting with comprehensive programs of recommended interventions in pilot ASPs. Non-academic hospitals have implemented interventions that are required by national guidelines and are expanding the program with other important interventions for a comprehensive ASP. Obviously the academic nature and larger size of academic hospitals support a more experimental, comprehensive approach, whereas non-academic hospitals seem to take a more practical approach by focusing on easily obtained gains first. Some existing processes can be reused as many activities that are now under the ASP umbrella are not necessarily new. For example, many hospitals have had remote consults with microbiologists in place for years. Also, this difference between academic and non-academic hospitals is strengthened by the fact that non-academic hospitals seem to have more difficulties in securing adequate resources for ASP.

When we combine maturity modelling with the findings of the current progress in ASP in hospitals included in our questionnaire, a number of conclusions can be drawn:

- In the state before ASP really starts, the null or $\mathrm{m}_{0}$ state of a maturity model, most Dutch hospitals already have a comprehensive antibiotic formulary and at least guidelines for treatment of infections, antibiotic therapy and prophylaxis. This is helpful as it can be a head start for implementing ASPs. In many other countries such an antibiotic formulary did not yet exist and is usually an important first ASP activity of the antibiotic team (van Limburg M, Köck R, Karreman J, Sinha B, de Jong N, Wentzel J, Friedrich A, Hendrix R, van Gemert-Pijnen J, "Towards an Implementation Strategy for Antibiotic/ Antimicrobial Stewardship: A Systematic Review", Under review).

- In the first stage of maturity, the initial phase, processes are ad-hoc and unorganised [11]. We found that hospitals are triggered by the SWAB vision document [9] and data from the questionnaire suggests that stakeholders are busy with a primary bundle of interventions that constitute an ASP. An

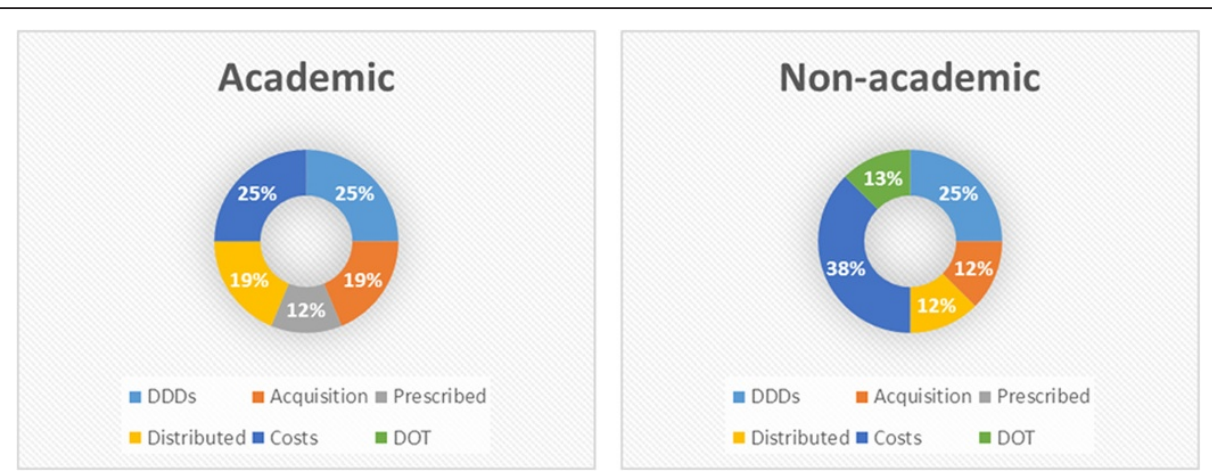

Figure 3 Preferences for data sources for benchmarking. ${ }^{*}$ ) DDDs: daily defined doses; DOT: days of therapy. 


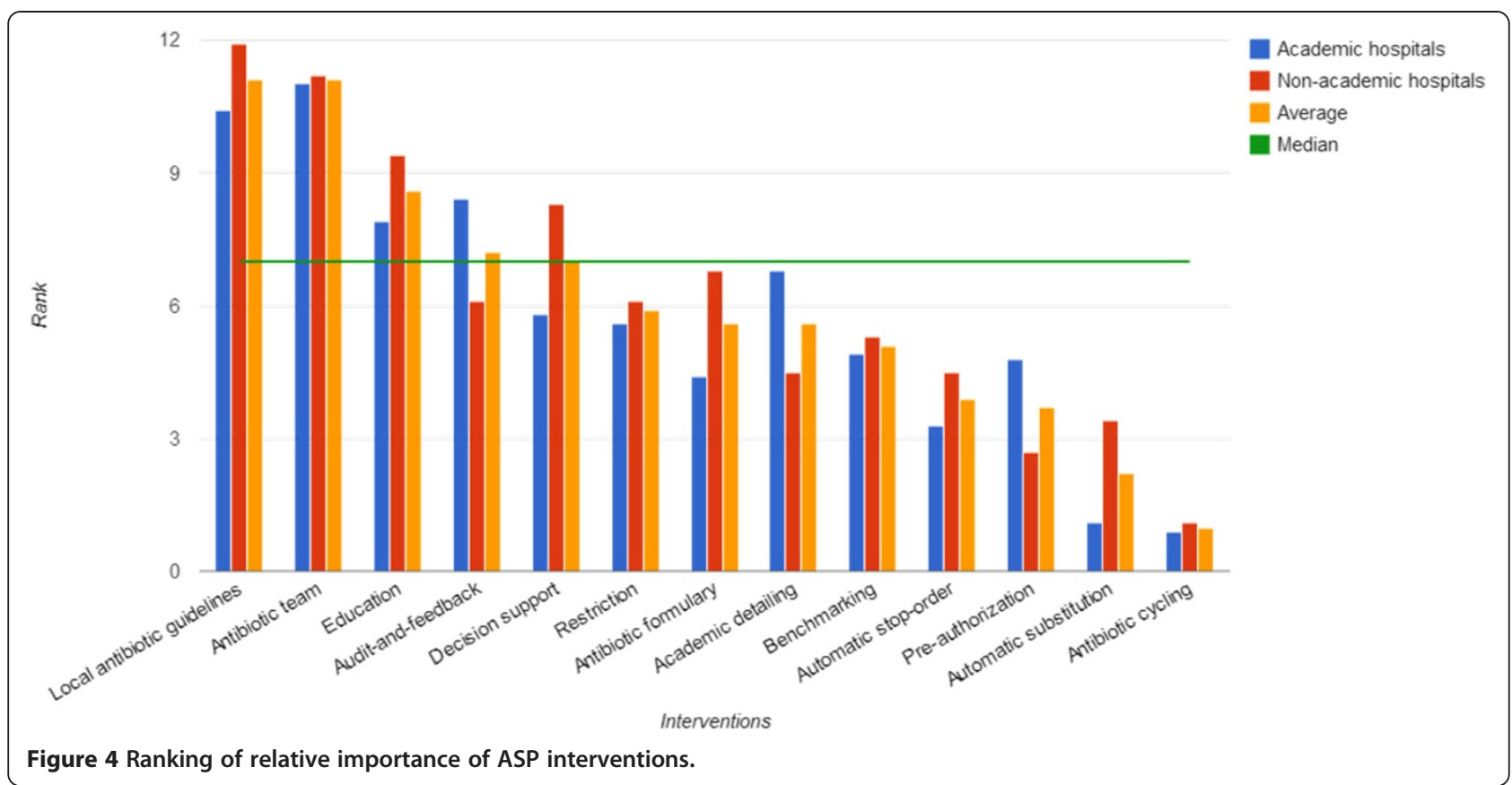

antibiotic team, adequate local antibiotic guidelines for ASP, educational activities and an audit-and-feedback intervention receive early attention and seem to be the first interventions to be implemented when hospitals start with ASP. However, it seems each intervention is implemented quite differently, according to local contexts and readily available means. In other words, interventions are implemented with a slight variation between them. For example, an intervention that seems rather straight-forward is an antibiotic team, however, the composition of an antibiotic team is already quite different in each hospital, depending on the available staff, and care focuses (children, trauma, etc.) in that hospital.

- The second stage of maturity, a managed state, is what regulatory documents aim for. There is a risk that the current proliferation of local ASPs and local variations between interventions in hospitals are unstandardized and will therefore be difficult to regulate, compare and manage. For example, how does a team with an ID physician relate to a team without? Timely guidelines that help standardisation are necessary. From a regulatory perspective, two scenarios are possible: a) allow proliferation and wait until a dominant design emerges - assuming that over time current ASPs will evolve into comparable programs or b) interact with hospitals and understand the local differences and anticipate with the regulations.

- Further stages of maturity, which is a measured and self-optimising state, is difficult to achieve for ASPs in their current state. Standardisation in processes and measurements are necessary to evaluate ASPs both internally and externally. We found that data collection for benchmarking can be done; however, as ASPs are currently novel and diverse between hospitals, this would be like comparing apples and oranges. Also, although there is evidence that ASPs show positive effects on antibiotic use and antibiotic resistance $[14,15]$, there is little or no standardisation in how effectiveness of ASPs is measured in terms of standardised outcomes or even methodologies [16,17]. That is also causing difficulties to compare (different) programs.

The assessment of the implementation of ASP can be expanded further by understanding the rationale and considerations local experts have based on expert-based recommendations in available regulatory documents. Patel et al. concluded that little guidance is offered on the practical aspects of implementing ASPs and that non-academic hospitals in the US need to overcome implementation issues by accounting for unique characteristics of their institutions [18]. Understanding these issues combined with these unique characteristics can eventually be used for parameters to customise local implementations of expert-based recommendations.

Earlier maturity assessments identified a set of structure indicators or characteristics that need to be present $[13,19]$. Prudence should be shown to assess the maturity of ASP implementation when using these structure indicators, as local experts may have valid reasons to deviate from expert recommendations. For example, a non-academic hospital may not have infectious disease 
physicians but a clinical microbiologist performing similar tasks. If available maturity assessments are strictly observed, this example hospital is missing an important structure indicator, while in practice the ASP was implemented differently from the ideal program as proposed and assessed by experts. Therefore, tallying whether expert-recommended interventions are present is not enough to capture the progress and maturity of ASP implementations.

By applying convenience sampling, we contacted respondents indirectly via infection control experts in the Dutch eastern border region and we depended on their willingness and involvement with ASP to find and persuade colleagues to partake. Due to the novelty of ASP, some experts declined participation as they claimed they were not sufficiently progressed with ASP initiatives yet. As a result, this study assessed only nineteen stakeholders from nine hospitals in the Dutch border region. Conclusions of this study do not reflect the entirety of Dutch hospitals. However, this was not the primary goal of the study, as we wanted to assess the implementation process of early ASP initiatives, for which the sample size sufficed. Another limitation was that by reducing the number of questions, not every intervention was assessed in high detail. We also omitted questions regarding microbiological diagnostics, infrastructure or tests and policy forming to keep the questionnaire at an acceptable length.

To keep the momentum going of ASP implementations and to improve the chances of success of these implementations, future research is necessary to obtain further insights into the rationale and issues that local experts consider during local implementations. What considerations did they have and why? At academic hospitals it would be interesting to assess what was learned with experimentation in pilot implementations. How did they arrive at the interventions as used in pilots and how will these pilots be scaled up? Case studies of these pilots can lead to concrete examples of potential and different implementations that can be used for advising other hospitals various configuration possibilities of ASPs. At non-academic hospitals, future research will be more focussed on practical issues in implementing ASP. What do local experts in non-academic hospitals consider in practice when implementing ASP? What are easily obtainable gains? What are barriers?

These different case studies, potential issues and rationale behind characteristics between interventions can be used as parameters that influence the configuration of to-be implemented ASPs. By inventorying all these parameters, local experts can be supported in customising and implementing an ASP that fits their hospital. Eventually, these parameters could be used to synthesise an implementation maturity toolkit. We plan to design a decision aid for experts in academic and non-academic hospitals that will generate a customised implementation advice for ASP fit for their local conditions.

\section{Conclusion}

Advising an ASP implementation is not straightforward. Experts who are tasked to introduce ASPs in their hospitals use expert-driven guidelines but need to transpose these guidelines to locally implemented interventions. This transposition leaves leeway for experimentation and considerations and leads to local differences in implemented intervention and ASPs. Progress can be made by assisting local experts with implementing ASPs in their hospitals. This assistance, however, needs to take into account that local conditions need to be translated into practical implementation advice and that a 'one-ASPfor-all' advice does not meet the needs of local experts. A bottom-up assessment with local experts can find parameters that influence local implementations of ASPs. These parameters can be used as input for a decision aid that generates a customised advice for a local implementation of an ASP.

\section{Additional file}

\section{Additional file 1: English translation of online questionnaire} "Maturity assessment of antibiotic stewardship programs".

\section{Competing interests}

The authors declare that they have no competing interest.

\section{Authors' contributions}

$M V L$ and JVG determined the study design and coordination. MvL prepared the questionnaire for data collection and performed all data collections and analyses. MvL interpreted the data and prepared the manuscript. JVG, BS and $J R L$ contributed to the manuscript. MvL and JVG critically revised the manuscript. All authors read and approved the final manuscript.

\section{Acknowledgements}

We would like to express our thanks to all ASP experts who contributed to the questionnaire, and thank H.C. Ossebaard, PhD, R.M.G. Hendrix, MD PhD, prof. A.W. Friedrich and prof. R. Sanderman for proofreading. We would also like to thank N. de Jong, J. Karreman and M.J. Wentzel for their help with the questionnaire.

\section{Financial support}

The studies presented in this article were financially supported by EurSafety Health-net, an European INTERREG IV-A major project. More project information is available at http://www.eursafety.eu.

\section{Author details}

${ }^{1}$ Department Psychology Health and Technology, Faculty Behavioral, Management and Social Sciences, University of Twente, Enschede, the Netherlands. ²Department of Medical Microbiology, University of Groningen, University Medical Center Groningen, Groningen, the Netherlands.

Received: 23 May 2014 Accepted: 10 September 2014

Published: 23 October 2014

\section{References}

1. World Health Organisation: WHO Global Strategy For Containment Of Antimicrobial Resistance. 2001 
2. European Union, Communication from the Commission to the European Parliament and the Council: Action Plan against the Rising Threats from Antimicrobial Resistance. 2011. http://ec.europa.eu/dgs/health_consumer/ docs/communication_amr_2011_748_en.pdf.

3. Monroe S, Polk R: Antimicrobial use and bacterial resistance. Curr Opin Microbiol 2000, 3(5):496-501.

4. Fishman N: Antimicrobial stewardship. Am J Infect Control 2006, 34(5):S55-S63.

5. MacDougall C, Polk R: Antimicrobial stewardship programs in health care systems. Clin Microbiol Rev 2005, 18(4):638.

6. Society for Healthcare Epidemiology of America, Infectious Diseases Society of America, Pediatric Infectious Diseases Society: Policy Statement on Antimicrobial Stewardship by the Society for Healthcare Epidemiology of America (SHEA), the Infectious Diseases Society of America (IDSA), and the Pediatric Infectious Diseases Society (PIDS). Infect Control Hosp Epidemiol 2012, 33(4):322-327.

7. Dellit TH, Owens RC, MCGowan JE, Gerding DN, Weinstein RA, Burke JP, Huskins WC, Paterson DL, Fishman NO, Carpenter CF, Brennan PJ, Billeter M, Hooton TM: Infectious Diseases Society of America and the Society for Healthcare Epidemiology of America Guidelines for Developing an Institutional Program to Enhance Antimicrobial Stewardship. Clin Infect Dis 2007, 44(2):159-177.

8. Allerberger F, Gareis R, Jindrák V, Struelens MJ: Antibiotic stewardship implementation in the EU: the way forward. Expert Rev Anti-Infect Ther 2009, 7(10):1175-1183.

9. SWAB: De Kwaliteit Van Het Antibioticabeleid In Nederland Advies Aangaande Het Restrictief Gebruik Van Antibiotica En Het Invoeren Van Antibioticateams In De Nederlandse Ziekenhuizen En In De Eerste Lijn. 2012. http://www.swab.nl/swab/cms3.nsf/uploads/5FD2BE2700E8B433 C1257A680028D9F0/\$FILE/visiedoc\%20SWAB\%20vs\%2021\%20junifinal.pdf.

10. Barlam TF: Antibiotic-stewardship practices at top academic centers throughout the United States and at hospitals throughout Massachusetts. Infect Control Hosp Epidemiol 2006, 27(7):695-703.

11. Paulk MC, Curtis B, Chrissis MB, Weber CV: Capability maturity model, version 1.1. Softw IEEE 1993, 10(4):18-27.

12. De Bruin T, Freeze R, Kaulkarni U, Rosemann M: Understanding the Main Phases of Developing a Maturity Assessment Model. 2005.

13. Burgmann $\mathrm{H}$, Janata $\mathrm{O}$, Allerberger $\mathrm{F}$, Frank $\mathrm{A}$ : Hospital antibiotic management in Austria-results of the $A B S$ maturity survey of the $A B S$ International group. Wien Klin Wochenschr 2008, 120(9-10):280-283.

14. Davey P, Brown E, Charani E, Fenelon L, Gould IM, Hartman G, Holmes A, Ramsay CR, Wiffen PJ, Wilcox M: Interventions to improve antibiotic prescribing practices for hospital inpatients. Cochrane Database Syst Rev 2013, 4(4):CD003543. doi:10.1002/14651858.CD003543.pub 16.

15. Kaki R, Elligsen M, Walker S, Simor A, Palmay L, Daneman N: Impact of antimicrobial stewardship in critical care: a systematic review. J Antimicrob Chemother 2011, 66(6):1223-1230.

16. Ramsay C, Brown E, Hartman G, Davey P: Room for improvement: a systematic review of the quality of evaluations of interventions to improve hospital antibiotic prescribing. J Antimicrob Chemother 2003, 52(5):764-771

17. McGowan JE Jr: Antimicrobial stewardship—the state of the art in 2011: focus on outcome and methods. Infect Control Hosp Epidemiol 2012, 33(4):331-337.

18. Patel D, MacDougall C: How to make antimicrobial stewardship work: practical considerations for hospitals of all sizes. Hosp Pharm 2010, 45(1):S10-S18

19. Buyle FM, Metz-Gercek S, Mechtler R, Kern WV, Robays H, Vogelaers D, Struelens MJ: Development and validation of potential structure indicators for evaluating antimicrobial stewardship programs in European hospitals. Eur J Clin Microbiol Infect Dis 2013, 32(9):1161-1170.

doi:10.1186/2047-2994-3-33

Cite this article as: van Limburg et al:: Evaluation of early

implementations of antibiotic stewardship program initiatives in nine Dutch hospitals. Antimicrobial Resistance and Infection Control 2014 3:33. 